\title{
Local Excitation of Magnetostatic Modes in YIG
}

\author{
Elisa Papa ${ }^{1}$, Stewart E. Barnes ${ }^{2}$, and Jean-Philippe Ansermet ${ }^{1}$ \\ ${ }^{1}$ Ecole Polytechnique Fédérale de Lausanne, IPMC, 1015 Lausanne, Switzerland \\ ${ }^{2}$ Physics Department, University of Miami, Coral Gables, 33124 FL USA
}

\begin{abstract}
Described are ferromagnetic resonance (FMR) studies of the long wavelength magnetostatic wave modes of a single crystal yttrium iron garnet (YIG) slab magnetized by an in-plane field. A resonant circuit comprising a coil, $50 \mu \mathrm{m}$ in diameter, is used to excite and detect the YIG microwave absorption spectrum. By changing its orientation and position over the sample surface it is possible to excite selectively different series of magnetostatic modes. The observed resonance fields are in good agreement with those calculated numerically from the Damon-Eshbach dispersion relation. The effect of heat currents on the magnetization dynamics in insulating magnets is explored. For a YIG crystal $10 \mathrm{~mm}$ in length, $2 \mathrm{~mm}$ in width and 25 micrometers in thickness, a temperature gradient of about $20 \mathrm{~K} / \mathrm{cm}$ is sufficient to suppress the high-order longitudinal magnetostatic modes.
\end{abstract}

Index Terms-Ferromagnetic resonance (FMR), magnetostatic modes, spin caloritronics, yttrium iron garnet (YIG).

\section{INTRODUCTION}

$\mathbf{T}$ HE microwave absorption spectrum of a yttrium iron garnet (YIG) $\left(\mathrm{Y}_{3} \mathrm{Fe}_{2}\left(\mathrm{FeO}_{4}\right)_{3}\right)$ slab has been extensively studied over the past decades. A variety of measuring techniques has been used. Experiments are often performed with a standard cavity, thereby irradiating the entire YIG sample by a uniform RF field. However, with the increasing interest in spatially resolved imaging and the local characterization of the magnetic excitations, local ferromagnetic resonance (FMR) measurement techniques have been quickly developed. Found in the literature [1]-[3] are many local FMR investigations performed by means of wire couplers or other local RF probes. Imaging of spin waves by Brillouin light scattering (BLS) spectroscopy has also been demonstrated [4]-[6] as well as a phase-sensitive BLS technique which further enables the detection of the phase structure of magnetostatic waves [7]. Other methods for local measurements of the dynamics of magnetization such as scanning Kerr imaging can also be found in the literature [8]. These observations can be accounted for with the theory of magnetostatic modes. The first theoretical analysis of magnetostatic waves in rectangular ferromagnetic films of infinite extent was presented by Damon and Eshbach in 1961 [9]. Refined models on the theory of magnetostatic wave modes were developed later in [10]-[12].

YIG has always attracted a lot of attention because of its low loss, making it a good model system [13], [14]. Several reports describing the temperature dependence of YIG magnetostatic modes can also be found [15]-[17]. However, to date, there is very little experimental work on the effect of temperature gradients on the microwave absorption spectrum of YIG slabs. Very recently, using a uniform RF field, Azevedo et al. [18] have studied the FMR of a YIG thin film subjected to a temperature gradient. Understanding the interplay between heat currents and the dynamics of magnetization is one of the main motivations

Manuscript received September 16, 2012; revised October 27, 2012; accepted November 12, 2012. Date of current version February 20, 2013. Corresponding author: J.-P. Ansermet (e-mail: jean-philippe.ansermet@epfl.ch).

Color versions of one or more of the figures in this paper are available online at http://ieeexplore.ieee.org.

Digital Object Identifier 10.1109/TMAG.2012.2229386 for the new branch of spintronics coined "spin caloritronics". This novel domain of research was triggered by the observation of the spin Seebeck effect in 2008 [19].

In the work described here, the long wavelength magnetostatic modes of a YIG slab are investigated by means of a local probe. It is shown that, depending on the position and orientation of the loop probe, different modes can be preferentially excited. These measurements are performed isothermally at room temperature. It is found that imposing a temperature gradient of the order of $20 \mathrm{~K} / \mathrm{cm}$ changes the spectrum drastically.

\section{EXPERIMENT}

Local ferromagnetic resonance measurements are carried out at $4 \mathrm{GHz}$ on a polished single crystal YIG slab $2 \mathrm{~mm} \times 10 \mathrm{~mm} \times \sim 25 \mu \mathrm{m}$ glued on a glass substrate 0.75 $\mathrm{mm}$ thick (provided by Ferrisphere). The field is applied in plane, parallel to the long side of the sample. A conventional field-modulation technique with a lock-in amplifier detection is employed. The measured absorption derivatives are then integrated to give the FMR spectra. Both the local excitation and detection are provided by a small probe (ordered from "ez SQUID", Sinn, Germany) consisting of a microstrip line short-circuited at one end by a copper coil and capacitively coupled to an SMA connector at the other end. The diameter of the coil, about $50 \mu \mathrm{m}$, determines the length scale of the exciting $\mathrm{RF}$ field $\left(\mathbf{h}_{\mathrm{rf}}\right)$. The distance between the coil and the sample surface is approximately $500 \mu \mathrm{m}$, in order to ensure good signal detection, but at the same time prevent large coupling. While the microwave probe resonates at around $7.25 \mathrm{GHz}$, in order to avoid an excessive coupling to the more intense modes, the FMR measurement is performed well off resonance at $4 \mathrm{GHz}$. The RF signal from a microwave source is sent to the local probe via the input port of a circulator. Changes in the amount of reflected power, probed by a crystal detector at the output port of the circulator, are measured while the applied magnetic dc field is scanned across the resonance.

By changing the position and the orientation of the probe relative to the sample, the coupling to the various FMR modes can be modified. Reports of similar studies can be found in the literature [20], but using a wire long compared to the sample. Fig. 1 shows the four different measurement configurations used in 


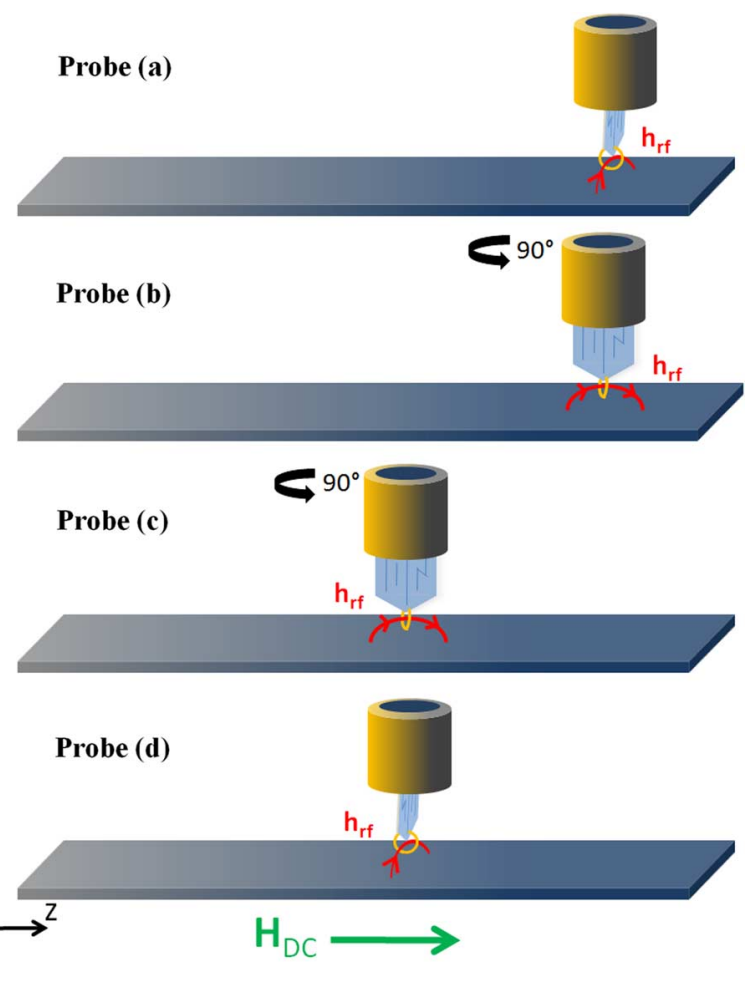

Fig. 1. Four different probe configurations used to locally measure FMR in a single crystal YIG slab $2 \mathrm{~mm} \times 10 \mathrm{~mm} \times \sim 25 \mu \mathrm{m}$. In (a) and (b) the probe is positioned near one end of the sample with the plane of the coil parallel and perpendicular to $\mathbf{H}_{\mathrm{DC}}$, respectively. In (c) and (d) the probe is in the middle of the sample with the plane of the coil perpendicular and parallel to $\mathbf{H}_{\mathrm{DC}}$, respectively. The external dc field is applied in plane in the $\mathrm{z}$ direction.

this work. In all four cases the loop probe is carefully centered with respect to the width of the sample. In configurations (a) and (b) the coil is positioned at about $1 / 4$ of the sample length with its plane parallel to the applied magnetic field $\left(\mathbf{H}_{\mathrm{DC}}\right)$ in (a) and perpendicular to $\mathbf{H}_{\mathrm{DC}}$ in (b). Therefore, in probe orientation (a), both RF field components $\left(\mathrm{h}_{\mathrm{rf}, \mathrm{x}}\right.$ and $\left.\mathrm{h}_{\mathrm{rf}, \mathrm{y}}\right)$ perpendicular to $\mathbf{H}_{\mathrm{DC}}$ can excite the magnetization, whereas in probe orientation (b) only the RF field component in the $\mathrm{x}$ direction $\left(\mathrm{h}_{\mathrm{rf}, \mathrm{x}}\right)$ is perpendicular to $\mathbf{H}_{\mathrm{DC}}$. In configurations (c) and (d) the probe is oriented as in (b) and (a) respectively, but it is positioned at the center of sample.

\section{RESUlTS AND DISCUSSION}

Figs. 2 and 3 show the absorption spectra measured with the previous four probe configurations by sweeping the applied field from saturation. The spectra are plotted for decreasing magnetic field going from left to right.

The FMR spectrum obtained with probe (a), in particular, presents a fine structure with a ratio of line spacing of the fine structure to that of the main structure equal to the ratio of sample length to width, suggesting excitation of long wavelength magnetostatic wave modes. Indeed, following the modeling described in the next section, the modes can be identified by the indices $\left(\mathrm{n}_{\mathrm{y}}, \mathrm{n}_{\mathrm{z}}\right)$, which denote the number of half wavelengths in the $\mathrm{y}$ (width) and $\mathrm{z}$ (length) directions, respectively.

Both of the spectra in Fig. 2 are measured with the probe located near one end of the sample. Odd and even $n$ modes are

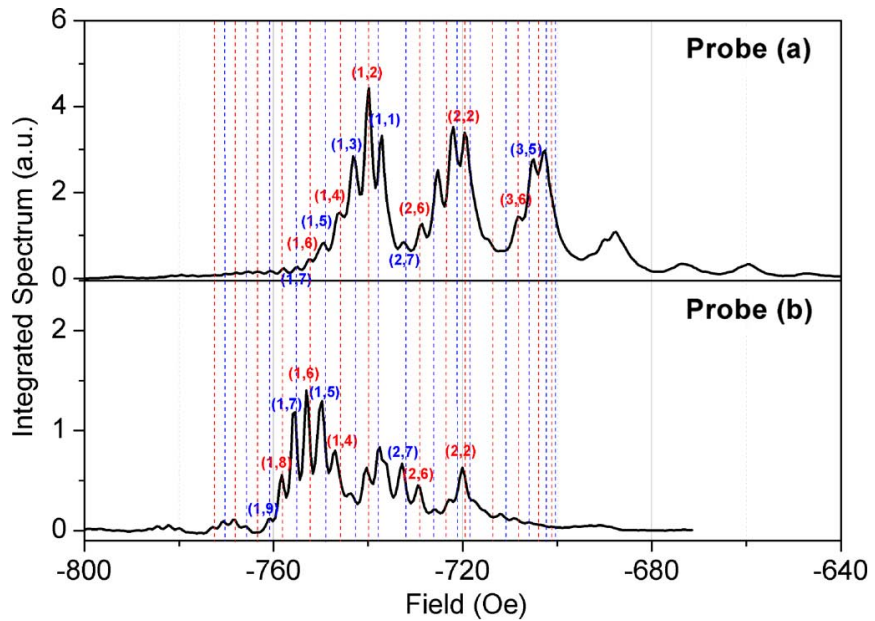

Fig. 2. Room temperature FMR absorption measured at $4 \mathrm{GHz}$ with probe configuration (a) for the top spectrum and probe configuration (b) for the bottom spectrum (see Fig. 1 for details of probe orientation and position). Spectra are plotted for decreasing field going from left to right. Dotted lines represent the resonance fields of the $\left(n_{y}, n_{z}\right)$ modes calculated from (1) in the text. Some of the identified resonance peaks are labelled by the associated indices $\left(\mathrm{n}_{\mathrm{y}}, \mathrm{n}_{\mathrm{z}}\right)$.

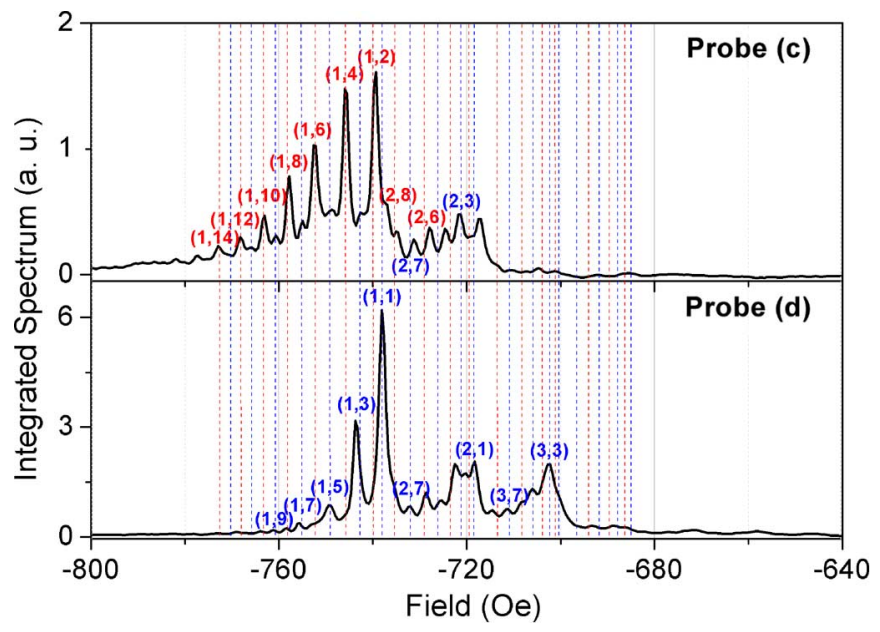

Fig. 3. Room temperature FMR absorption measured at $4 \mathrm{GHz}$ with probe configuration (c) for the top spectrum and probe configuration (d) for the bottom spectrum (see Fig. 1 for details of probe orientation and position). Spectra are plotted for decreasing field going from left to right. Dotted lines represent the resonance fields of the $\left(\mathrm{n}_{\mathrm{y}}, \mathrm{n}_{\mathrm{z}}\right)$ modes calculated from (1) in the text. Some of the identified resonance peaks are labelled by the associated indices $\left(n_{y}, n_{z}\right)$.

selected. In the top spectrum, taken with probe configuration (a), $\mathbf{h}_{\mathrm{rf}}$ is fully perpendicular to $\mathbf{H}_{\mathrm{DC}}$. The RF field component $\mathrm{h}_{\mathrm{rf}, \mathrm{x}}$ has the right symmetry to excite even $\mathrm{n}_{\mathrm{y}}$ modes, while $h_{r f, y}$ excites odd $n_{y}$ modes. Here, since the location of the coil is off the symmetry plane of the sample, the RF field can couple to modes with any $n_{z}$.

The bottom spectrum (Fig. 2) is instead collected with probe configuration (b). In this case, only the component $h_{r f, x}$ excites modes. It has the wrong symmetry to couple to transverse modes (in the y direction). Presumably, the $\mathrm{n}_{\mathrm{y}}=2$ is excited nonetheless because of the imperfect position and orientation of the probe. In this configuration, higher $\mathrm{n}_{\mathrm{z}}$ order modes $\left(\mathrm{n}_{\mathrm{z}}>4\right)$ are clearly seen, since the favored modes must have a node of $\mathrm{h}_{\mathrm{rf}, \mathrm{x}}$ underneath the probe. 
Both spectra in Fig. 3 are taken with the probe positioned in the middle of the sample and configuration (c) excites even $\mathrm{n}_{\mathrm{z}}$ modes, whereas configuration (d) excites odd $\mathrm{n}_{\mathrm{z}}$ modes. In configuration (c), $h_{r f, x}$ has, in fact, a node at the position of the coil. Therefore, in this case magnetostatic wave modes with a node in the middle of the sample, i.e., with even $n_{\mathrm{z}}$ values are favored. The resonance peaks of modes $(1,2),(1,4),(1,6),(1,8)$, $(1,10),(1,12),(1,14)$ with decreasing amplitude for increasing order are indeed easily identified. In this configuration, only the $\mathrm{n}_{\mathrm{y}}=1$ modes are well excited as in case (b).

With the probe in configuration (d), the RF field is again perpendicular to $\mathbf{H}_{\mathrm{DC}}$ as in configuration (a), so it can fully contribute to exciting the magnetization. Since the RF field is maximum at the middle of the sample it couples strongly to magnetostatic modes with odd $n_{\mathrm{z}}$ values. Thus, at the bottom of Fig. 3 , the modes $(1,1),(1,3),(1,5),(1,7)$ and $(1,9)$ can be recognized. Since both $h_{r f, x}$ and $h_{r f, y}$ contribute to the excitation along the $\mathrm{y}$ direction, $\mathrm{n}_{\mathrm{y}}$ can take both even and odd values.

From all the recorded spectra it can be observed that the modes of the lowest indices are favored. This is due to the length scale of the RF field inhomogeneity that is determined by the gap of about $500 \mu \mathrm{m}$ between the probe and the sample. Finally, it should be noted that the magnetostatic wave modes excited in the $\mathrm{z}$ direction for $\mathrm{n}_{\mathrm{y}}>1$ tend to get closer to each other and the peaks are hardly resolved.

\section{THEORY}

Magnetostatic wave modes are magnetic excitations which arise from the classical dipole-dipole interaction (exchange is negligible). They can be interpreted as the normal modes of vibration of the magnetization vector which is a function of position within the sample and time. The length scale of this class of magnetic excitation is of the same order of magnitude as the linear dimensions of the sample under study. Therefore, the characteristic wavelengths of these wave-like modes are small enough to satisfy the magnetostatic approximation and sufficiently large, in most cases, to neglect exchange [11].

The dispersion relation for these resonant magnetostatic wave modes propagating in a rectangular thin slab of infinite dimensions was calculated for the first time by Damon and Eshbach [9]. By simultaneously solving Maxwell's equations in the magnetostatic limit and the equation of motion of the magnetization (neglecting losses), the following characteristic equation for magnetostatic wave modes was derived:

$$
\begin{aligned}
& \left(1+\eta^{2}\right)+2\left|\left(1+\eta^{2}\right)^{\frac{1}{2}}\right|\left(-\frac{1+\eta^{2}+\kappa}{1+\kappa}\right)^{\frac{1}{2}} \\
& \times(1+\kappa) \cot \left[\left|k_{y}\right| s\left(-\frac{1+\eta^{2}+\kappa}{1+\kappa}\right)^{\frac{1}{2}}\right] \\
& +(1+\kappa)^{2}\left(\frac{1+\eta^{2}+\kappa}{1+\kappa}\right)-\nu^{2}=0
\end{aligned}
$$

where

$$
\begin{gathered}
\eta=\frac{k_{z}}{k_{y}}, \quad \kappa=\frac{\Omega_{H}}{\left(\Omega_{H}^{2}-\Omega^{2}\right)}, \quad \nu=\frac{\Omega}{\left(\Omega_{H}^{2}-\Omega^{2}\right)} \\
\Omega_{H}=\frac{H_{i}}{4 \pi M_{S}}, \quad \Omega=\frac{\omega}{4 \pi \gamma M_{S}}, \quad k_{y}=\frac{n_{y} \pi}{l_{y}}, \quad k_{z}=\frac{n_{z} \pi}{l_{z}} .
\end{gathered}
$$

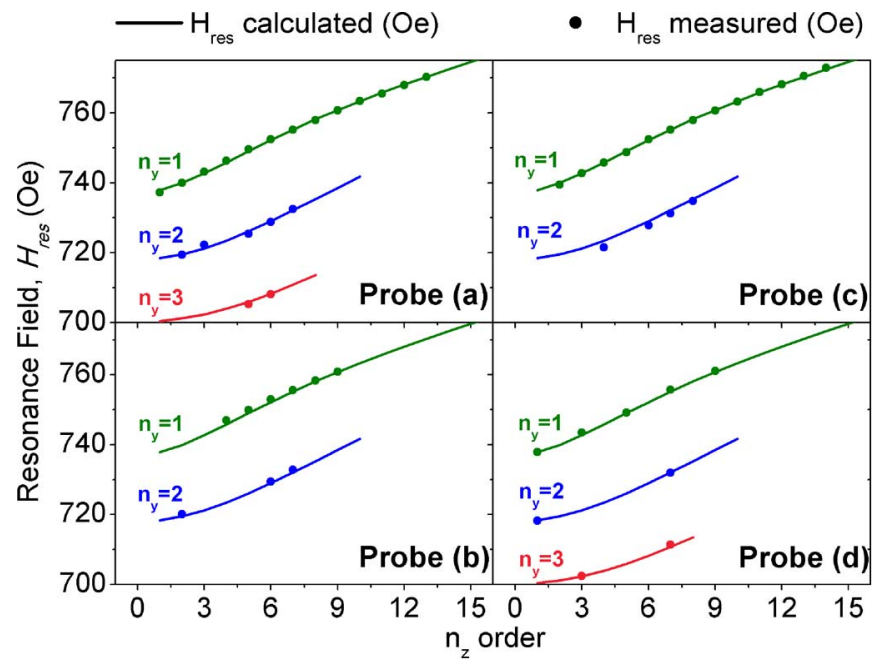

Fig. 4. Resonance fields of the $\left(\mathrm{n}_{\mathrm{y}}, \mathrm{n}_{\mathrm{z}}\right)$ magnetostatic wave modes excited with four different probe configurations as indicated in the plots (see Fig. 1 for details of probe orientation and position in each configuration). Curves are calculated from (1) and the points are taken from the resonance field values in the measured spectra.

Here $M_{S}$ is the saturation magnetization, $\omega$ is the FMR angular frequency, $\mathrm{H}_{\mathrm{i}}$ is the internal magnetic field, $\gamma$ is the gyromagnetic ratio, $l_{y}$ and $l_{z}$ are the width and length of the sample, $s$ is the thickness and $n_{\mathrm{y}}$ and $n_{\mathrm{z}}$ are the mode orders. The values of $k_{y}$ and $k_{z}$ are assumed to be quantized due to the boundary condition of zero RF magnetization at the sample edges. The observed modes are then stationary waves that result in resonances at discrete field values. Since $s \ll l_{y, z}$, this implicit equation of $H_{i}\left(k_{y}, k_{z}\right)$ for a given $\omega$ can be used to determine the resonance fields of the magnetostatic modes in a film of finite dimensions.

Fig. 4 shows the calculated and measured resonance fields of the excited magnetostatic modes as a function of mode number. In order to attain good agreement between measured and computed resonance fields, the parameters $4 \pi M_{S}=1936.5 \mathrm{G}$, $(\gamma / 2 \pi)=2.8 \mathrm{MHz} / \mathrm{Oe}$ and $s=24.7 \mu \mathrm{m}$ are used in the calculation. This value of $4 \pi M_{S}$ gives the best fit, but it is substantially larger than the bulk value around $1780 \mathrm{G}$. This accounts for other anisotropy fields not reflected by other terms in (1). Hence the value of $M_{S}$ used here can be taken as the value of an effective magnetization $M_{S}^{\prime}$ that includes the total of the anisotropy effects [21].

\section{FMR With TEMPERATURE GRADIENT}

The effect of a temperature gradient on the magnetostatic mode spectrum of YIG has been investigated. The specimen is fixed over two Peltier elements as shown in Fig. 5 by using a thermal sticker in order to optimize the thermal contact. A temperature gradient of about $22 \mathrm{~K} / \mathrm{cm}$ is applied to the sample by heating one end to $313 \mathrm{~K}\left(\mathrm{~T}_{1}\right)$ and cooling down the other to $291 \mathrm{~K}\left(\mathrm{~T}_{2}\right)$. FMR measurements are performed at the cold end of the sample with the coil oriented and positioned as in configuration (a) illustrated in Fig. 1. The temperature gradient changes the microwave absorption spectrum as shown in Fig. 5. There is in fact a clear and distinct simplification of the spectrum as compared to the room temperature FMR spectra reported in Figs. 2 and 3. The high order longitudinal modes that appear at room 

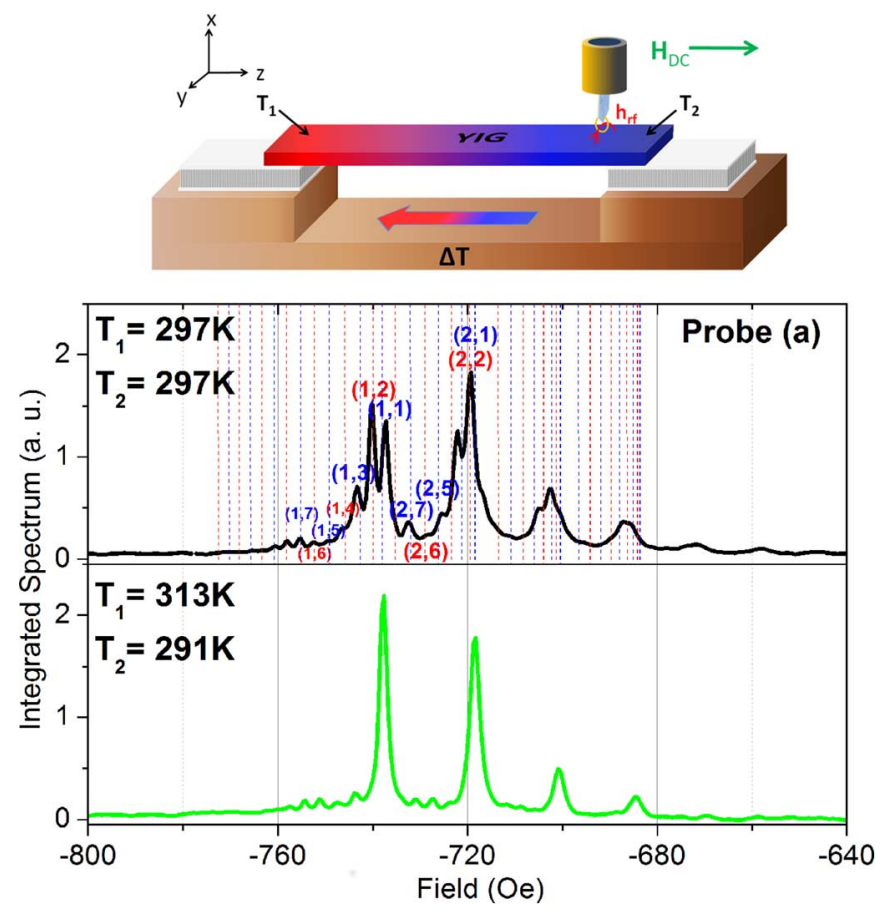

Fig. 5. FMR spectra of a single crystal YIG slab $2 \mathrm{~mm} \times 10 \mathrm{~mm} \times \sim 25 \mu \mathrm{m}$ measured at room temperature (top spectrum) and in presence of a $\Delta \mathrm{T}=22 \mathrm{~K}$ between the sample ends in the $\mathrm{z}$ direction (bottom spectrum).

temperature are suppressed in presence of the temperature bias and only the transverse modes of low $\mathrm{n}_{\mathrm{z}}$ order are effectively excited. However, it is difficult to assess the exact $n_{z}$ index value of these peaks due to the small spacing $(<2 \mathrm{G})$ between the $\mathrm{n}_{\mathrm{z}}=1$ and $\mathrm{n}_{\mathrm{z}}=2$ modes, especially for $\mathrm{n}_{\mathrm{y}}>1$. More work has to be carried out on samples of smaller dimensions. Greater spacing between the different $n_{\mathrm{z}}$ modes can facilitate the assignment of the resonance peaks and can therefore help determine whether certain modes are enhanced by the temperature gradient or whether the pinning of the higher order modes disappears.

No such change in spectrum is detected when the measurement is performed at the hot end of the sample. In this case, the fine structure of the spectrum, associated to longitudinal modes, remains. Moreover, for a set temperature gradient, the observed peaks appear at different resonance fields as the probe is scanned over the sample surface.

The simplification of the spectrum in presence of temperature gradient measured at the cold end of the sample is in contrast with the result reported by Rezende and Azevedo in 1992 which shows that the number of excited modes in a YIG film increases when the sample is subjected to a field gradient [22].

Further analysis is still required to understand whether the temperature gradient is more than just a perturbation to the system. If so, the excited modes and their associated wave vector component $\mathrm{k}_{\mathrm{z}}$ may become fundamentally different.

\section{CONCLUSION}

The magnetostatic modes of a single crystal of YIG are excited with a small coil of $50 \mu \mathrm{m}$ located $500 \mu \mathrm{m}$ above the sample surface. Mode quantization is clearly exhibited demonstrating these are extended throughout the sample. The orientation and location of the coil relative to the sample, i.e., in the middle or near the ends, lead to the preferential excitation of the various modes. The observations agree well with the Damon-Eshbach model.

A YIG slab of $10 \mathrm{~mm}$ in length is mounted on Peltier elements to create a temperature gradient along the length of the sample. A mere $20 \mathrm{~K}$ temperature difference is sufficient to cause a significant simplification of the excitation spectrum with the suppression of the high order longitudinal modes.

\section{ACKNOWLEDGMENT}

This work was supported by the Sino-Swiss Science and Technology Cooperation Program (SSSTC) under Grant IZL CZ2 123999 and the Germany priority program DFG SS 1538 under Grant AN 762/1-1.

\section{REFERENCES}

[1] T. An, N. Ohnishi, T. Eguchi, Y. Hasegawa, and P. Kabos, "Local excitation of ferromagnetic resonance and its spatially resolved detection with an open-ended radio-frequency probe," IEEE Magn. Lett, vol. 1, p. 3500104,2010

[2] J. Barak, R. Ruppin, and J. T. Suss, "Selective excitation of magnetostatic modes of a thin film," Phys. Lett. A, vol. 108, no. 8, pp. 423-425, Apr. 1985.

[3] L. K. Brundle and N. J. Freedman, "Magnetostatic surface waves on a y.i.g. slab," Electron. Lett., vol. 4, no. 7, p. 132, 1968.

[4] A. Azevedo and S. M. Rezende, "Spatial distribution of magnetostatic modes in a thin YIG slab," J. Magn. Magn. Mater., vol. 104-107, no. 0, pt. 2, pp. 1039-1040, Feb. 1992.

[5] M. Bauer, O. Büttner, S. O. Demokritov, B. Hillebrands, V. Grimalsky, Y. Rapoport, and A. N. Slavin, "Observation of spatiotemporal selffocusing of spin waves in magnetic films," Phys. Rev. Lett., vol. 81, no. 17 , pp. 3769-3772, Oct. 1998.

[6] O. Büttner, M. Bauer, S. O. Demokritov, B. Hillebrands, Y. S. Kivshar V. Grimalsky, Y. Rapoport, and A. N. Slavin, "Linear and non-linear diffraction of dipolar spin waves in yttrium iron garnet films observed by space- and time-resolved Brillouin light scattering," Phys. Rev. B, vol. 61, pp. 11576-11587, May 2000.

[7] A. A. Serga, T. Schneider, B. Hillebrands, S. O. Demokritov, and M. P. Kostylev, "Phase-sensitive Brillouin light scattering spectroscopy from spin-wave packets," Appl. Phys. Lett., vol. 89, no. 6, pp. 063506-063506-3, Aug. 2006.

[8] S. Tamaru, J. A. Bain, R. J. M. van de Veerdonk, T. M. Crawford, M. Covington, and M. H. Kryder, "Measurement of magnetostatic mode excitation and relaxation in permalloy films using scanning Kerr imaging," Phys. Rev. B, vol. 70, no. 10, p. 104416, Sep. 2004.

[9] R. W. Damon and J. R. Eshbach, "Magnetostatic modes of a ferromagnetic slab," J. Phys. Chem. Solids, vol. 19, pp. 308-320, May 1961.

[10] B. A. Kalinikos and A. N. Slavin, "Theory of dipole-exchange spin wave spectrum for ferromagnetic films with mixed exchange boundary conditions," J. Phys. C: Solid State Phys., vol. 19, pp. 7013-7033, Dec. 1986.

[11] M. J. Hurben and C. E. Patton, "Theory of magnetostatic waves for in-plane magnetized isotropic films," J. Magn. Magn. Mater., vol. 139, pp. 263-291, Jan. 1995.

[12] D. D. Stancil and A. Prabhakar, Spin Waves: Theory and Applications, 1st ed. New York: Springer, 2009, pp. 139-168.

[13] A. A. Serga, A. V. Chumak, and B. Hillebrands, "YIG magnonics," $J$ Phys. D: Appl. Phys., vol. 43, no. 26, p. 264002, Jul. 2010.

[14] M. Sparks, B. R. Tittmann, J. E. Mee, and C. Newkirk, "Ferromagnetic resonance in epitaxial garnet thin films," J. Appl. Phys., vol. 40, no. 3, pp. 1518-1525, Mar. 1969.

[15] I. Laulicht, J. T. Suss, and J. Barak, "The temperature dependence of the ferromagnetic and paramagnetic resonance spectra in thin yttriumiron-garnet films," J. Appl. Phys., vol. 70, no. 4, pp. 2251-2258, Aug. 1991.

[16] J. S. Baek, S. Y. Ha, W. Y. Lim, and S. H. Lee, "Magnetostatic wave study of YIG films grown by the LPE method," Phys. Stat. Sol., vol. 201, no. 8, pp. 1806-1809, 2004.

[17] M. Kaack, S. Jun, S. A. Nikitov, and J. Pelzl, "Magnetostatic spin wave modes excitation in yttrium-iron-garnet film under various temperatures," J. Magn. Magn. Mater., vol. 204, no. 1-2, pp. 90-100, Sep. 1999. 
[18] G. L. da Silva, L. H. Vilela-Leao, S. M. Rezende, and A. Azevedo, "Spin current injection by spin Seebeck and spin pumping effects in yttrium iron garnet/Pt structures," J. Appl. Phys., vol. 111, no. 7, p. 07C513, 2012.

[19] K. Uchida, S. Takahashi, K. Harii, J. Ieda, W. Koshibae, K. Ando, S. Maekawa, and E. Saitoh, "Observation of the spin Seebeck effect," Nature, vol. 455 , no. 7214 , pp. $778-781$, Oct. 2008.

[20] J. Barak and U. Lachish, "Study of the excitation of magnetostatic modes in yttrium-iron-garnet films by a microstrip line," J. Appl. Phys., vol. 65 , no. 4, pp. 1652-1658, Feb. 1989.

[21] J. A. Duncan, B. E. Storey, A. O. Tooke, and A. P. Cracknell, "Magnetostatic modes observed in thin single-crystal yttrium iron garnet at Q-band frequencies," J. Phys. C: Solid State Phys., vol. 13, no. 10, pp. 2079-2095, Apr. 1980
[22] S. M. Rezende and A. Azevedo, "Dipolar narrowing of ferromagnetic resonance lines," Phys. Rev. B, vol. 44, no. 13, pp. 7062-7065, Oct. 1991.

[23] B. E. Storey, A. O. Tooke, A. P. Cracknell, and J. A. Przystawa, "The determination of the frequencies of magnetostatic modes in rectangular thin films of ferrimagnetic yttrium iron garnet," J. Phys. C: Solid State Phys., vol. 10, no. 6, pp. 875-888, Mar. 1977. 